Z Rheumatol 2014 · 73:215-216

DOI 10.1007/s00393-013-1241-7

Online publiziert: 28. März 2014

c) Springer-Verlag Berlin Heidelberg 2014

J. Wollenhaupt ${ }^{1} \cdot$ A. Zink ${ }^{2}$

${ }^{1}$ Klinik für Rheumatologie und klinische Immunologie, Schön Klinik Hamburg Eilbek, Hamburg

${ }^{2}$ Deutsches Rheuma-Forschungszentrum (DRFZ), Berlin

\title{
Rheuma im höheren Lebensalter
}

me. Die im Alter veränderte Pharmakokinetik und die möglichen Auswirkungen auf bestehende Komorbiditäten verlangen einen vorsichtigeren Einsatz und zusätzliche Kontrollmaßnahmen. Die geringere Versorgung älterer Patienten mit Biologika muss daher keine schlechtere Versorgung dieser Patienten bedeuten, sondern ist auch Ausdruck der Beachtung altersbedingter Veränderungen, beispielsweise des erhöhten Infektionsrisikos und der Begleitmedikationen. Allerdings müssen auch und gerade beim älteren Patienten Nutzen und Risiken der Therapiealternativen sorgfältig abgewogen werden. So kann die (bei Älteren häufigere) Gabe von Glukokortikoiden das Infektionsrisiko nachhaltiger erhöhen als der Einsatz von Biologika.

Die Versorgung des alten, multimorbiden Patienten verlangt in besonderer Weise nach einer multimodalen, interdisziplinären und patientenorientierten Versorgung. Christoph Fiehn stellt in seinem Übersichtsbeitrag dar, dass die in der Geriatrie etablierte systematische Erfassung psychosozialer Aspekte, der Polypharmazie oder der Compliance durchaus beispielgebend auch für die rheumatologische Regelversorgung sein kann. Der zunehmende Mangel an Hausärzten und die steigende Komplexität fachspezifischer Therapieschemata verlangen nach echten interdisziplinären Strukturen, in denen Spezialisten unterschiedlicher Fachrichtungen miteinander interagieren. Dies ist v. a. dort möglich, wo die Interdisziplinarität Strukturmerkmal ist, wie in fächerübergreifenden Praxisgemeinschaften, medizinischen Versorgungszentren, \$116b-Ambulanzen oder im Rahmen der stationären multimodalen Komplexbe- handlung. Auch die Einbindung nichtärztlicher Berufsgruppen ist dort leichter zu realisieren als in der Einzelpraxis oder dem nicht spezialisierten Krankenhaus.

Infektionen stellen für ältere Patienten ein besonderes Risiko dar. Aufgrund der im Alter verringerten Aktivität des Immunsystems, der gesteigerten Organvulnerabilität und bereits bestehender Vorschäden der Organe verlaufen Infektionskrankheiten häufig schwerer. In ihrem Beitrag stellen Christian Kneitz, Anja Strangfeld und Klaus Krüger die Möglichkeiten dar, bereits im Vorfeld einer immunsuppressiven Therapie älterer $\mathrm{Pa}$ tienten geeignete Maßnahmen zu ergreifen, um das Risiko infektiöser Komplikationen zu verringern. Im Fall einer manifesten Infektion sind zudem beim älteren Patienten bei der Therapie einige Besonderheiten zu beachten, die ebenfalls erläutert werden.

Eine besondere Herausforderung ist die Schmerztherapie des älteren Menschen. Naturgemäß nehmen Arthrose und Osteoporose mit ihren Folgen mit zunehmendem Lebensalter zu. Auch bestehen bei entzündlich-rheumatischen Erkrankungen nicht selten strukturelle Schäden, die zu sekundären Schmerzen führen. Gleichzeitig führen Änderungen der Pharmakokinetik, Einschränkungen der hepatischen bzw. renalen Elimination und bestehende Begleiterkrankungen zu einer Verringerung der Einsatzmöglichkeiten schmerzsenkender Pharmaka. Die Diskussion der kardiovaskulären Nebenwirkungsprofile nichtsteroidaler Antirheumatika oder die Sturzrisiken bei Einsatz zentralwirksamer Analgetika sind weitere Beispiele für die Problematik, gerade älteren Patienten mit muskuloske- 
lettalen Schmerzen angemessen helfen $\mathrm{zu}$ können. Franziska Luttosch und Christoph Baerwald erläutern die Dimension dieses Problembereichs und therapeutische Lösungsmöglichkeiten.

Eine angemessene Sicherheit der Behandlung spielt demnach bei älteren und hochbetagten Patienten mit rheumatischen und muskuloskelettalen Erkrankungen eine zentrale Rolle. Gleichzeitig kann heute als gesichert gelten, dass eine ausreichende entzündungshemmende Behandlung entzündlich-rheumatischer Erkrankungen nicht nur möglich, sondern vorteilhaft ist. Klaus Krüger, Anja Strangfeld und Christian Kneitz stellen dar, dass die verbesserte Kontrolle chronischer Entzündungsvorgänge durch Einsatz von Immunsuppressiva bei älteren Patienten krankheitsimmanente Risiken senkt, ohne dass therapeutische Risiken unangemessen zunehmen. Ihr Beitrag zur Sicherheit der Rheumatherapie im Alter und der damit verbundenen Grundsätze zu Einsatzmöglichkeiten und Überwachung der Therapie schließt diesen Themenschwerpunkt ab.

Ihre

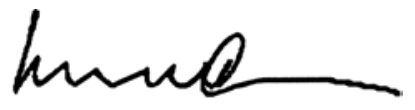

Jürgen Wollenhaupt

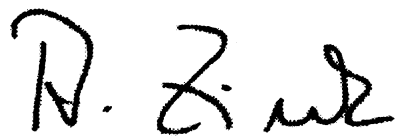

Angela Zink

\section{Korrespondenzadressen}

Prof. Dr. J. Wollenhaupt

Klinik für Rheumatologie und klinische Immunologie, Schön Klinik Hamburg Eilbek Dehnhaide 120, 22081 Hamburg wollenhaupt@rheumatologikum.de

\section{Prof. Dr. A. Zink}

Deutsches Rheuma-Forschungszentrum (DRFZ) Charitéplatz 1, 10117 Berlin

zink@drfz.de

Interessenkonflikt. J. Wollenhaupt und A. Zink geben an, dass kein Interessenkonflikt besteht.

\section{J. Köbberling}

\section{Diagnoseirrtum, Diagnose- fehler, Befunderhebungsfehler}

Bewertungen und Vermeidungsstrategien

Verlag Versicherungswirtschaft 2013, 1. Auflage, 181 S., (ISBN 3899527704), Taschenbuch, 39.00 EUR

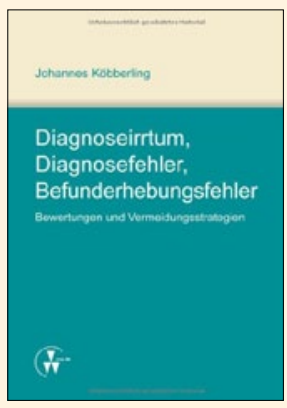

Ärzte müssen sich in Ihrer Berufsausübung zunehmend mit Vorwürfen über tatsächliche oder vermeintliche Fehler bei diagnostischen Maßnahmen und damit zusammenhängenden Haf-

tungsfragen befassen. Dies führt nicht selten zu großer Verunsicherung. Aus verschiedenen Gründen nehmen Verfahren, in denen Ärzten oder Kliniken Behandlungsfehler vorgeworfen werden, deutlich zu. Medienberichte über die moderne Medizin führen zu Fehleinschätzungen über das medizinisch Machbare und zu einer steigenden Erwartungshaltung bei Patienten. Die breiten Darstellungen von Leitlinien, die von jedermann im Internet aufrufbar sind, können leicht zu einer Fehleinschätzung der Verbindlichkeit von medizinischen Standards im Einzelfall führen. Komplikationen und Misserfolge werden ohne kritische Hinterfragung auf Behandlungsfehler zurückgeführt. Hinzu kommen steigende Erwartungen an einen möglichen Schadenersatz, eine gegenüber früher deutlich verminderte Hemmschwelle für Klagen und in vielen Fällen ein fehlendes Prozessrisiko durch bestehende Rechtsschutzversicherungen oder Prozessfinanzierungen. Fast ein Viertel aller Vorwürfe über Behandlungsfehler beziehen sich auf Diagnosefehler. Den meisten Ärzten sind die rechtlichen Aspekte, die sich im Zusammenhang mit möglichen Diagnosefehlern ergeben, nur unzureichend bekannt.

Mit dem vorliegenden Buch soll hier eine Lücke geschlossen werden. Die beiden Kapitel über "Diagnoseirrtum" und "Diagnosefehler" beginnen jeweils mit den Definitionen und mit den wichtigen Fragen der Abgrenzung voneinander. Während ein "noch verständlicher" Diagnoseirrtum nicht zu einer Haftung führt, ist ein nicht mehr verständlicher Diagnosefehler haftungsbegründend, wenn er zu einem Schaden bei dem Patienten führt. Diese in der Rechtsprechung entwickelten und in das Patientenrechtegesetz vom Februar 2013 übernommenen Definitionen lassen jeweils breite Deutungs- und Ermessensspielräume zu. Dies war Anlass für die Erstellung der Fallsammlung aus Gutachten oder Bescheiden der Gutachterkommission für ärztliche Behandlungsfehler und aus gerichtlichen Urteilen. Alle aufgeführten 54 Beispiele sind authentisch und mit Aktenzeichen versehen.

Ausführlich wird in einem gesonderten Kapitel auf die dritte Kategorie, den „Befunderhebungsfehler" eingegangen, der dazu führen kann, dass ein einfacher Diagnosefehler zu einem groben Behandlungsfehler mit Beweislastumkehr wird. Dieses komplizierte Konstrukt mit mehrstufiger Beweiswürdigung ist ebenfalls in das Patientenrechtegesetz aufgenommen worden.

Das sehr kompakte Kompendium ist bei der Abklärung von Behandlungsfehlern für Juristen, Ärzte und Patienten ein unverzichtbarer Ratgeber. Ärzte erhalten wertvolle Empfehlungen für das richtige Verhalten bei Vorliegen eines vermeintlichen Behandlungsfehlers.

M. Broglie (Wiesbaden) 